\title{
Kommunale Akteure verstehen: Vorgeschichte der Arbeit in Kommunen beachten
}

\author{
Jochen Eckart, Elke Häußler, Thorsten Erl, Monika Gonser \& Jan Riel
}

\section{Herausforderungen bei der Analyse kommunaler Akteure in Reallaboren}

Kommunen spielen in Reallaboren und in der transdisziplinären und transformativen Forschung allgemein oft eine zentrale Rolle. Aufbau und Organisation einer Kommune sind durchaus komplex und können von jeweils ortsspezifischen Besonderheiten geprägt sein. Insofern ist ein besseres Verständnis von Kommunen und insbesondere den einzelnen Akteuren innerhalb einer Kommune (Fachverwaltung, Verwaltungsspitze, politische Entscheidungsträger) von maßgeblicher Bedeutung für das Gelingen der Zusammenarbeit in solchen Projekten.

Für das bessere Verständnis der kommunalen Akteure in Reallaboren und in anderen transdisziplinären und transformativen Forschungsvorhaben bietet sich das Instrument der Akteursanalyse an (Eckart et al. 2018). Die Akteursanalyse hat sich aus Ansätzen der Politikfeldanalyse (Blum und Schubert 2011), des Strategic Management (Freeman 1984) sowie der Partizipationsforschung (Reed et al. 2009; Zimmermann 2006) entwickelt. Auch im Forschungskontext wird die Akteursanalyse bereits angewandt (Butterworth et al. 2011). Sie dient als Instrument, um die Einbindung der Praxisakteure in transdisziplinären und transformativen Forschungsvorhaben im Detail zu planen. Im Rahmen der Akteursanalyse sind die für die Forschungs-, Praxis- und Bildungsziele eines Vorhabens erforderlichen Akteure (Beecroft et al. 2018) zu identifizieren. Die Akteursanalyse befasst sich zudem mit den Erwartungen der Akteure darüber, wie und in welchem Umfang sie sich an einem Vorhaben beteiligen können oder sollen sowie mit den Möglichkeiten und Grenzen der Akteure. Damit eine Akteursanalyse gelingen kann und in der Folge die Zusammenarbeit in einem Projekt fruchtbar verläuft, ist es wichtig, den Besonderheiten der analysierten Akteure Rechnung zu tragen und nicht mit verkürztem Blick an die Analyse heranzugehen. Es ist also, mit anderen Worten, ein Bewusstsein nötig für mögliche ,blinde Flecken', die die Qualität einer Akteursanalyse beeinträchtigen könnten. 
Basierend auf Erfahrungen aus den vom Ministerium für Wissenschaft, Forschung und Kunst Baden-Württemberg (MWK) seit 2015 geförderten 14 Reallaboren der Förderlinien „Reallabore“ und „Reallabore Stadt" (im Folgenden BaWü-Labs) ${ }^{1}$ und bezogen auf den Akteur Kommune wird dargestellt, inwiefern ,blinde Flecken' existieren können, also Wissenslücken auf Seiten der Wissenschaftler(innen) über die Funktionsweise von und die Arbeit mit verschiedenen kommunalen Akteuren. Diese ,blinden Flecken' können in der Akteursanalyse sowie in der eigentlichen Forschungsarbeit zu empfindlichen Komplikationen führen - sei es, weil nicht die richtigen Ansprechpartner gefunden, weil vorgegebene Entscheidungswege nicht beachtet oder weil ohnehin vorbelastete Beziehungen zwischen Akteuren aus Unkenntnis überstrapaziert werden.

Diesen ,blinden Flecken' sind in diesem Buch drei Kurzbeiträge gewidmet: Ein Beitrag beschreibt die Verteilung von Wissensbeständen innerhalb von Kommunen und inwiefern Kommunen nicht nur als ein (homogener) Ansprechpartner (miss)zu verstehen sind (Erl et al. 2019 in diesem Buch). Ein Beitrag stellt die komplexen Entscheidungswege innerhalb kommunaler Strukturen dar, deren Kenntnis für das Gelingen der Zusammenarbeit von besonderer Bedeutung ist (Gonser et al. 2019 in diesem Buch). Der vorliegende Beitrag widmet sich der Vorgeschichte kommunaler Akteure und zeigt, warum es wichtig ist, sich mit dieser auseinanderzusetzen.

\section{Kontinuierliches Arbeiten in der Kommune}

Die Arbeit in Reallaboren oder in anderen transdisziplinären oder transformativen Forschungsvorhaben ist durch deren Projektcharakter mit einem klaren Anfangsund Endpunkt versehen. Die Arbeit der kommunalen Akteure zeichnet sich hingegen häufig durch ein kontinuierliches Arbeiten vor Ort an einem Thema aus. Thematische Arbeit ist daher aus Sicht der kommunalen Akteure meist kein einmaliges Projekt ohne Bezüge zur Vergangenheit oder zur Zukunft, sondern durch ein zeitliches und organisatorisches Vor-, Neben-, Mit- und Nacheinander geprägt (Streich 2005). Daher ist auch die Mitarbeit in Reallaboren und in verwandten Projekten aus kommunaler Sicht in vorherige und zukünftige Aktivitäten und Projekte eingebunden.

Zwei Teilaspekte sind hierbei zu unterscheiden: Die Kontinuität der Arbeit in einem Thema sowie die Kontinuität der Beziehung zwischen den beteiligten Akteuren. In den Kommunen gibt es bei den meisten Themen bereits einen historischen Vorlauf, an den sich die Akteure aus der Kommune bzw. in der Öffentlichkeit erinnern und worauf sie Bezug nehmen. Viele Themen sind daher in der

1 Für Informationen zu den beiden Förderlinien siehe http://www.reallabore-bw.de (zugegriffen am 20.05.2019). 
Einschätzung und dem Handeln vorgeprägt. Zudem ist das Verhältnis der handelnden Akteure auch durch Vorgeschichten geformt. So kann das für eine konstruktive Zusammenarbeit erforderliche Vertrauen zwischen den Wissenschaftler(inne)n und kommunalen Akteuren nicht innerhalb von Wochen oder wenigen Monaten entstehen, sondern benötigt eine längere Zeit und kann durch vergangene Interaktionen beeinflusst sein. Wenn solche Vorgeschichten negiert werden, entstehen ,blinde Flecken` in der Akteursanalyse.

\section{Die Vorgeschichte als Herausforderung für die Arbeit in und mit Kommunen}

Für die Arbeit in transdisziplinären und transformativen Projekten können sich Hürden ergeben, wenn diese thematisch sensible Punkte oder historisch vorbelastete Orte tangieren. In Themen, in denen hingegen positive Erfahrungen gemacht wurden, sehen sie sich eher mit hohen Erwartungen konfrontiert.

In der Kommune vorbelastete Themen (, heiße Eisen', ,Tabus'), werden von kommunalen Akteuren mit Vorsicht behandelt oder gar gänzlich gemieden. Dadurch kann es zu einem erhöhten Arbeitsaufwand durch zusätzlich erforderliche Kommunikationsprozesse oder zu Verzögerungen beim Projektfortschritt kommen. Dabei können Konflikte, die ihren Ursprung nicht in dem aktuellen Projekt selbst haben, die Zusammenarbeit mit den kommunalen Akteuren in diesem Projekt aber dennoch beeinflussen.

Im Reallabor Nachhaltige Transformation der Textilwirtschaft am Standort Dietenheim (,BaWü-Lab Dietenheim zieht an“) war die Kommunalverwaltung zeitweisen Vorwürfen von Fehlentscheidungen im Hinblick auf ein anderes, vom Reallabor unabhängiges, Projekt vor Ort ausgesetzt. Es entstand für die wissenschaftliche Seite der nicht zu belegende Eindruck, dass man daher seitens der Kommune zu dieser Zeit ,auf Nummer sicher' gehen wollte. So geriet die Planung einer Reallabor-Veranstaltung ins Stocken und konnte erst nach einer Aussprache erfolgreich fortgesetzt werden.

Im Reallabor Asylsuchende in der Rhein-Neckar-Region (,,BaWü-Lab Asylsuchende“) (s. auch Steckbrief im Anhang zu diesem Buch) sollten Fragen der dezentralen Unterbringung von Geflüchteten in einzelnen Stadt- und Ortsteilen wissenschaftlich untersucht werden. Zeitgleich waren die Kommunen auf der sehr konkreten Suche nach Gebäuden und Flächen, die sich zur Unterbringung oder zum Bau von Unterbringungen von Geflüchteten eignen würden. Der kommunale Suchprozess wurde von teils überaus kritischen Diskussionen unter den Bürger(inne)n begleitet. Dieser externe Konflikt wirkte sich auf den Ablauf des Reallabors aus, da die Kommunen teilweise eher zögerlich dem Forschungsanliegen zustimmten. So bestand wohl die Sorge, dass durch das Agieren mehrerer Akteure oder eine möglicherweise unklare Kommunikation der Ziele des Reallabors noch mehr Aufruhr oder möglicherweise Missverständnisse in die 
öffentliche Debatte eingebracht werden könnten. Um diesen Befürchtungen zu begegnen, wurden die einzelnen Forschungsschritte in enger Kommunikation mit den Kommunen vorgenommen und Kommunikationsprozesse detailliert abgesprochen.

Zudem können sich historische Konflikte auf die Zusammenarbeit mit weiteren Praxisakteuren in der Kommune auswirken und zu Verzögerungen oder Hindernissen im Projektverlauf führen.

Im Reallabor GO Karlsruhe - Partizipative Forschung für den Fußgängerverkehr (,,BaWü-Lab GO Karlsruhe“) bestand in einem Stadtteil ein Konflikt durch vorherige fehlgeschlagene Partizipationsaktivitäten. Die Ergebnisse einer weiteren externen Forscher(innen)gruppe trafen in einem Beteiligungsprozess auf starke Vorbehalte seitens der bürgerschaftlichen Akteure vor Ort. Die ablehnende Haltung war jedoch nicht durch die Wissenschaftler(innen) selbst verursacht, sondern begründete sich durch weitere externe Konflikte in einem Neubaugebiet des Stadtteils und durch die Unzufriedenheit mit der kommunalen Verwaltung. Aufgrund dieser historischen Konflikte zwischen externer Forscher(innen)gruppe, kommunaler Verwaltung und bürgerschaftlichen Akteuren verlief die auf das Reallabor bezogene Partizipation in diesem Stadtteil deutlich langsamer und weniger erfolgreich als in anderen Stadtteilen ohne negative Vorgeschichte.

Weiterhin können die Beziehungen zwischen den verschiedenen Akteuren in einem Projekt durch die Vorgeschichte geprägt sein (Eckart et al. 2018; Butterworth et al. 2011). Eine wichtige Grundlage für eine erfolgreiche Zusammenarbeit zwischen den Wissenschaftler(inne)n und den kommunalen Akteuren ist das gegenseitige Vertrauen. Es kann verschieden stark belastbare Vertrauensverhältnisse zwischen den Akteuren geben, die sich auf den Gestaltungsspielraum der Zusammenarbeit in einem Projekt auswirken können.

In manchen BaWü-Labs gab es bestehende und gut funktionierende Arbeitsbeziehungen zwischen den Wissenschaftler(inne)n und den kommunalen Akteuren, die auf eine Zeit vor dem Reallabor zurückgingen. Dadurch bestand bereits ein gegenseitiges Vertrauen und damit verbunden die Bereitschaft der kommunalen Akteure, auch über das gewohnte Verwaltungshandeln hinausgehende Aktivitäten anzugehen. Ist das Vertrauensverhältnis hingegen durch frühere Erfahrungen belastet, kann dies ein deutliches Hindernis in der Zusammenarbeit bilden.

Im Fall des BaWü-Labs GO Karlsruhe war die Konstellation insofern günstig, als einer der Wissenschaftler zuvor viele Jahre in einem der Fachämter in der Kommune beschäftigt war. Die Entscheidungsstrukturen sowie die inhaltlichen Positionen von Verwaltungsakteuren waren daher zum einen den Wissenschaftler(inne)n bekannt, zum anderen konnten aber auch die Akteure der Verwaltung die Anfragen der Wissenschaftler(innen) von vorneherein besser einschätzen. Die fachliche Position und die Arbeitsweise der Wissenschaftler(innen) waren den Mitarbeitenden der Verwaltung ebenfalls bekannt, so dass in verschiedenen Fällen keine besondere Überzeugungsarbeit zu leisten war. Eine solche Ausgangslage ist sicherlich selten. Unabhängig davon erscheinen der Weg über bestehende Verbindungen und das Aufbauen auf bereits vorhandene Vertrauensverhältnisse sinnvoll. 
In Projekten entstehen neue Arbeitsbeziehungen zwischen Wissenschaftler(inne)n und kommunalen Akteuren. Hier ist besondere Aufmerksamkeit auf den Vertrauensaufbau zu legen, um eine produktive Arbeitsbeziehung zu schaffen. Dafür erforderlich sind insbesondere die gegenseitige Akzeptanz der Fachkenntnis sowie ein offener, fairer Umgang miteinander.

Im BaWü-Lab Asylsuchende waren mit Blick auf die Zusammenarbeit mit einigen Akteuren zunächst der gemeinsame Besuch einer Fachveranstaltung sinnvoll, gefolgt von der Konzeption und Durchführung einer Einzelveranstaltung, hin zur Konzeption einer Veranstaltungsreihe und eines ersten experimentellen Projektsettings etc., um schrittweise die Möglichkeit zu geben, das Vertrauen auszuweiten. Auch die ,Lieferung' zunächst einseitiger Beiträge wie z. B. die Aufbereitung von Fachinformationen, die nur bedingt mit dem konkreten Reallabor zu tun hatten, für den kommunalen Akteur aber gerade relevant waren, konnte Vertrauen schaffen.

\section{Berücksichtigung der Vorgeschichte in der Akteursanalyse}

Die Vorgeschichte der Arbeit in einem Thema sowie des Verhältnisses zwischen den Akteuren ist zu analysieren, da sich durch deren Unkenntnis Schwierigkeiten im Ablauf eines Projekts ergeben können. Bewährt hat sich im Rahmen der Akteursanalyse, diese nicht allein auf die gegenwärtigen Rahmenbedingungen der Akteure zu konzentrieren, sondern auch die Vorgeschichte des Themas sowie der Beziehung der Akteure in den Blick zu nehmen. Mögliche Fragen für eine Akteursanalyse, die diesen , blinden Fleck' adressieren, sind:

- Welche Ergebnisse oder Erfahrungen aus vorangegangenen Prozessen oder Projekten im sozialräumlichen und thematischen Gebiet des Projekts gibt es?

- Wie etabliert sind die Arbeitsbeziehungen und das Vertrauen zwischen den Akteuren aus vorherigen Prozessen oder Projekten?

Diese Fragen können im Rahmen von Interviews mit kommunalen Insidern geklärt werden. Dies kann weiter durch Interviews mit ausgewählten externen Praxisakteuren ergänzt werden, die mit dem entsprechenden kommunalen ,Feld“ vertraut sind und eine Einschätzung, von außen‘ bieten können.

\section{Literatur}

Beecroft, R., Trenks, H., Rhodius, R., Benighaus, C., \& Parodi, O. (2018). Reallabore als Rahmen transformativer und transdisziplinärer Forschung: Ziele und Designprinzipien. In R. Defila \& A. Di Giulio (Hrsg.), Transdisziplinär und transformativ forschen. Eine Methodensammlung. (S. 75-100). Wiesbaden: Springer VS. doi: 10.1007/978-3-65821530-9 4.

Blum, S., \& Schubert, K. (2011). Politikfeldanalyse. Wiesbaden: Springer VS. 
Butterworth, J., McIntyre, P., \& da Silva Wells, C. (Hrsg.). (2011). Switch in the City: Putting Urban Water Management to the Test. Den Haag: IRC International Water and Sanitation Centre.

Eckart, J., Ley, A., Häußler, E., \& Erl, Th. (2018). Leitfragen für die Gestaltung von Partizipationsprozessen in Reallaboren. In R. Defila \& A. Di Giulio (Hrsg.), Transdisziplinär und transformativ forschen. Eine Methodensammlung (S. 105-135). Wiesbaden: Springer VS. doi: 10.1007/978-3-658-21530-9_6.

Freeman, R. E. (1984). Strategic Management: A Stakeholder Approach. Boston: Pitman.

Reed, M. S., Graves, A., Dandy, N., Posthumus, H., Hubacek, K., \& Morris, J. (2009). Who's in and why? A typology of stakeholder analysis methods for natural resource management. Journal of Environmental Management, 90, (S. 1933-1949).

Streich, B. (2005). Stadtplanung in der Wissensgesellschaft. Ein Handbuch. Wiesbaden: VS Verlag für Sozialwissenschaften.

Zimmermann, A. (2006). Instrumente zur Akteursanalyse - 10 Bausteine für die partizipative Gestaltung von Kooperationssystemen. Eschborn: Deutsche Gesellschaft für Technische Zusammenarbeit (GTZ).

\section{Die zwei weiteren Beiträge in diesem Buch zu ,blinden Flecken ‘}

Erl, Th., Gonser, M., Eckart, J., Häußler, E., \& Riel, J. (2019). Kommunale Akteure verstehen: Verteilte Wissensbestände in Kommunen. In R. Defila \& A. Di Giulio (Hrsg.), Transdisziplinär und transformativ forschen, Band 2. Eine Methodensammlung (S. 139145). Wiesbaden: Springer VS.

Gonser, M., Riel, J., Eckart, J., Erl, Th., \& Häußler, E. (2019). Kommunale Akteure verstehen: Komplexe Entscheidungswege in Kommunen. In R. Defila \& A. Di Giulio (Hrsg.), Transdisziplinär und transformativ forschen, Band 2. Eine Methodensammlung (S. 85-91). Wiesbaden: Springer VS.

Open Access Dieses Kapitel wird unter der Creative Commons Namensnennung 4.0 International Lizenz (http://creativecommons.org/licenses/by/4.0/deed.de) veröffentlicht, welche die Nutzung, Vervielfältigung, Bearbeitung, Verbreitung und Wiedergabe in jeglichem Medium und Format erlaubt, sofern Sie den/die ursprünglichen Autor(en) und die Quelle ordnungsgemäß nennen, einen Link zur Creative Commons Lizenz beifügen und angeben, ob Änderungen vorgenommen wurden.

Die in diesem Kapitel enthaltenen Bilder und sonstiges Drittmaterial unterliegen ebenfalls der genannten Creative Commons Lizenz, sofern sich aus der Abbildungslegende nichts anderes ergibt. Sofern das betreffende Material nicht unter der genannten Creative Commons Lizenz steht und die betreffende Handlung nicht nach gesetzlichen Vorschriften erlaubt ist, ist für die oben aufgeführten Weiterverwendungen des Materials die Einwilligung des jeweiligen Rechteinhabers einzuholen.

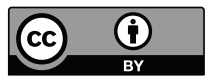

